\title{
Heterosis Studies in Sunflower (Helianthus annuus L.)
}

\author{
D. Ramaraju, A. B. Rajguru, H. J. Rajput* and R. D. Nimbalkar \\ Division of Botany, College of Agriculture, Pune, Maharashtra, India \\ *Corresponding author
}

\begin{tabular}{|l|}
\hline Ke y w o r d s \\
Sunflower, \\
Heterosis, \\
Helianthus annuus \\
\hline Article Info \\
\hline $\begin{array}{l}\text { Accepted: } \\
\text { 20 August } 2019 \\
\text { Available Online: } \\
\text { 10 September } 2019\end{array}$ \\
\hline
\end{tabular}

\section{A B S T R A C T}

The present investigation entitled "Heterosis studies in sunflower (Helianthus annuus L.) was undertaken to estimate the heterosis for seed yield and its components in sunflower. The four CMS lines and eight restorer lines were crossed in line x tester model to produce 32 hybrids during Summer-2017-18 and these 32 hybrids along with two checks were evaluated for days to 50 percent flowering, day to maturity, plant height $(\mathrm{cm})$, head diameter $(\mathrm{cm})$, seed filling percentage $(\%), 100$ seed weight $(\mathrm{g})$, volume weight $(\mathrm{g} / 100 \mathrm{ml})$, hull content $(\%)$, seed yield per plant $(\mathrm{g})$ and oil content $(\%)$. The magnitude of heterosis over the check Phule Raviraj and MDSFH-411 for most of the characters in the present study were highly appreciable. Among the hybrid viz. CMS-89 x NSH-312 showed the highest significant heterosis for seed yield and its components over the checks Phule Raviraj and MDSFH-411. From the investigation the following hybrids were found promising i.e. CMS-343 $\mathrm{x}$ PSMOO-108, CMS-89-1 x NSH-312, CMS-62 x AKSF-12 and CMS-343 x MRHA-2 which showed high per se performance comprising significant standard heterosis for seed yield along with significant heterosis in desirable direction for most of the yield attributes.

\section{Introduction}

The sunflower (Helianthus annuus L.) is important oil seed crop. The genus Helianthus is grown as a crop for its edible oil and edible fruits. Under this genus there are 67 species, cultivated in different forms like oilseed crops, ornamental, landscaping etc and is extensively grown in Russia, USA, China and India. Sunflower is one of the most important oilseed crops in India and ranks fifth after soybean, mustard, groundnut and sesame as edible oil source. In India during 2016-17, sunflower was cultivated on an area of 3.44 lakh hectares with a production of 2.40 lakh tones. The average yield of $699 \mathrm{~kg} / \mathrm{ha}$ was one of the lowest in world (Anonymous, 2017). It is rich source of edible oil (40 to $45 \%$ ) and is considered as good quality oil from health point of view, due to high concentration of polyunsaturated fatty acids (55 to $60 \%$ linoleic acid, 25 to $30 \%$ oleic acid), which are known to reduce the risk of coronary disease by reducing the cholesterol in blood plasma. The 
importance of sunflower as an oilseed crop in India is of very recent origin and date backs to four decades.

Sunflower is a native of North America but commercialization of the plant took place in Russia. In India, as an oilseed crop, it was introduced in 1969, prior to which it was used as an ornamental plant. Commercial cultivation in India started in 1972 in few hectares land in southern states because of wider adaptability, photoperiod insensitivity, short duration, high yield. A land mark in sunflower breeding was discovery of cytoplasmic male sterility by Leclereq (1969) and restorer genes in wild type sunflower cultivar (Kinman, 1970). In sunflower, the cytoplasmic male sterility (CMS) system, which involves the use of CMS line (A), maintainer line (B) and fertility restorer line (R) has allowed breeders to exploit heterosis and heterosis breeding

In heterosis breeding programme, large number of hybrids are produced and evaluated to exploit hybrid vigor, which usually requires more resources and manpower. It is possible to select the parental lines based on their genetic diversity status to affect limited crosses with good success, if there is a relationship between heterosis for yield and genetic diversity.

\section{Materials and Methods}

The present studies on heterosis in sunflower (Helianthus annuus L.) were conducted during Kharif 2018 at Agriculture Botany Farm, College of Agriculture, Pune. The crossing programme carried out in line $\mathrm{x}$ tester design during Summer-2017-18. The experimental material for study included 4 CMS lines (CMS-234, CMS-62, CMS-89-1, CMS-343) and 8 restore lines (NSH-312, PSCIM-165, PSMOO-108, PSFRMM-140, MRHA-2, AKSF-345, AKSF-12, CSFR-99) to produce
32 hybrids along with 2 checks (Phule Raviraj, MDSFH-411) in RBD design. The parental lines used for crossing was collected from the Sunflower Physiologist, ZARS, Solapur. The heterosis of these 32 hybrids were evaluated and compared with checks.

\section{Results and Discussion}

Maximum negative heterosis was considered beneficial for days to 50 per cent flowering, days to maturity and plant height. For successful heterosis breeding programme in any crop, there are two important strategies involved i.e. presence of significant heterotic effect in the hybrids and feasibility of hybrid seed production. Sunflower is highly cross pollinated in nature; hence it offers tremendous scope for commercial exploitation of heterosis using cyto-restorer system. (Madrap and Makne 1993), Sugoor R K, (1992) and Gangappa et al.,(1997). Though heterotic vigour could be exploited by development of hybrids but commercialization of hybrids depends on the superiority over the standard checks. In other words, the standard heterosis of the newly developed hybrids is of prime importance for its adoption by farmers.

Generally the per se performance of the parents is found to be closely related with high heterotic hybrids. The magnitude of heterosis over the check Phule Raviraj and MDSFH-411 for most of the characters in the present study were highly appreciable.

Among the hybrid viz. CMS-343 x PSMOO108 showed the highest significant heterosis for days to 50 per cent flowering (-13.41, $10.40)$, days to maturity $(-8.30,-5.48)$ and volume weight $(23.54,26.47)$ while CMS-89 $\mathrm{x}$ NSH-312 for days to 50 per cent flowering $(-10.06,-6.94)$, days to maturity $(-6.86,-4.09)$, head diameter $(2.86,20.32)$, seed filling per cent $(10.60,12.86)$ and hull content(-15.39,11.90). 
Table.1 Per cent heterosis for ten different characters in sunflower hybrids

\begin{tabular}{|c|c|c|c|c|c|c|c|c|c|}
\hline \multirow[t]{2}{*}{$\begin{array}{l}\text { Sr. } \\
\text { no }\end{array}$} & \multirow[t]{2}{*}{ Hybrids } & \multicolumn{2}{|c|}{$\begin{array}{c}\text { Days to 50\% } \\
\text { Flowering (days) }\end{array}$} & \multicolumn{2}{|c|}{$\begin{array}{l}\text { Days to } \\
\text { Maturity } \\
\text { (days) }\end{array}$} & \multicolumn{2}{|c|}{$\begin{array}{c}\text { Plant } \\
\text { Height }(\mathbf{c m})\end{array}$} & \multicolumn{2}{|c|}{$\begin{array}{c}\text { Head } \\
\text { diameter }(\mathbf{c m})\end{array}$} \\
\hline & & $\mathrm{SC} 1$ & $\mathrm{SC} 2$ & $\mathrm{SC} 1$ & SC2 & $\mathrm{SC} 1$ & $\mathrm{SC} 2$ & SC1 & SC2 \\
\hline 1 & CMS-234 X NSH-312 & -3.35 & 0.00 & -2.89 & 0.00 & -7.15 & $-14.44 * *$ & 6.40 & 5.98 \\
\hline 2 & CMS-234XPSCIM165 & -0.56 & 2.89 & -0.72 & 2.23 & -8.43 & $-15.62^{* * *}$ & 4.00 & 3.59 \\
\hline 3 & CMS-234 X PSMOO-108 & $-7.26 * *$ & -4.05 & $-4.33 *$ & -1.49 & -6.93 & $-14.24 * *$ & 0.80 & 0.40 \\
\hline 4 & CMS-234 X PSFRMM-140 & -5.03 & -1.73 & $-3.97 *$ & -1.12 & -6.85 & $-14.16^{* * *}$ & -1.20 & -1.59 \\
\hline 5 & CMS-234 X MRHA-2 & $-6.70 *$ & -3.47 & $-4.33 *$ & -1.49 & 0.09 & -7.77 & 2.00 & 1.59 \\
\hline 6 & CMS-234 X AKSF-345 & 2.79 & $6.36^{*}$ & 1.08 & $4.09^{*}$ & -2.83 & $-10.45^{*}$ & 9.20 & 8.76 \\
\hline 7 & CMS-234 X AKSF-12 & -2.23 & 1.16 & -1.44 & 1.49 & 5.69 & -2.60 & 0.80 & 0.40 \\
\hline 8 & CMS-234 X CSFR-99 & -1.68 & 1.73 & -1.44 & 1.49 & -0.17 & -8.01 & $15.60^{* *}$ & $15.14 * *$ \\
\hline 9 & CMS-62 X NSH-312 & $-7.82 * *$ & -4.62 & $-5.78 * *$ & -2.97 & 3.34 & -4.77 & 1.20 & 0.80 \\
\hline 10 & CMS-62 X PSCIM-165 & $-10.06^{* *}$ & $-6.94 *$ & $-6.86^{* *}$ & $-4.09 *$ & 9.69 & 1.07 & 2.00 & 1.59 \\
\hline 11 & CMS-62 X PSMOO-108 & -0.66 & 2.89 & -0.36 & 2.60 & 1.67 & -6.31 & 5.60 & 5.18 \\
\hline 12 & CMS-62 X PSFRMM-140 & -4.47 & -1.16 & -2.89 & 0.00 & 9.33 & 0.75 & -3.20 & -3.59 \\
\hline 13 & CMS-62 X MRHA-2 & $-8.94 *$ & $-5.78 *$ & $-6.14 * *$ & -3.35 & 7.75 & -0.71 & 5.60 & 5.18 \\
\hline 14 & CMS-62 X AKSF-345 & -3.91 & -0.58 & -2.53 & 0.37 & 4.84 & -3.39 & 5.60 & 5.18 \\
\hline 15 & CMS-62 X AKSF-12 & $-7.26 * *$ & -4.05 & $-5.78 * *$ & -2.97 & $12.59 *$ & 3.75 & 2.00 & 1.59 \\
\hline 16 & CMS-62 X CSFR-99 & -4.47 & -1.16 & $-3.61 *$ & -0.74 & 3.49 & -4.69 & $13.60 *$ & $13.15^{*}$ \\
\hline 17 & CMS-343 X NSH-312 & $-10.61 * *$ & $-7.51 * *$ & $-7.58 * *$ & $-4.83 *$ & 7.06 & -1.34 & $14.00^{*}$ & $13.55^{*}$ \\
\hline 18 & CMS-343 X PSCIM-165 & $-8.94 * *$ & $-5.78^{*}$ & -6.14 & -3.35 & -7.11 & -1.30 & -7.60 & -7.97 \\
\hline 19 & CMS-343 X PSMOO-108 & $-13.41 * *$ & $-10.40^{* *}$ & $-8.30 * *$ & $-5.58 * *$ & -1.63 & -9.35 & $-18.40 * *$ & $-18.73 * *$ \\
\hline 20 & CMS-343 X PSFRMM-140 & 1.17 & 4.62 & 0.72 & $3.72 *$ & 0.04 & -7.81 & $-16.40 * *$ & $-16.73 * *$ \\
\hline 21 & CMS-343 X MRHA-2 & $-10.06 * *$ & $-6.94 *$ & $-7.22 * *$ & $-4.46^{*}$ & -7.66 & $-14.91 * *$ & -9.20 & -9.56 \\
\hline 22 & CMS-343 X AKSF-345 & $-8.38^{* *}$ & -5.20 & $-5.78 * *$ & -2.97 & 10.10 & 1.46 & $-12.80 *$ & $12.35^{*}$ \\
\hline 23 & CMS-343 X AKSF-12 & -3.91 & -0.58 & -2.53 & 0.37 & 8.13 & -0.36 & $16.40^{* *}$ & $15.94 * *$ \\
\hline 24 & CMS-343 X CSFR-99 & $-8.94 * *$ & $-5.78 *$ & $-5.78 * *$ & -2.97 & $13.66^{*}$ & 4.73 & $20.80^{* *}$ & $20.32 * *$ \\
\hline 25 & CMS-89-1 X NSH- 312 & $-10.06 * *$ & $-6.94 *$ & $-6.86 * *$ & $-4.09 *$ & -1.71 & -9.43 & 20.80 ** & $20.32 * *$ \\
\hline 26 & CMS-89-1 X PSCIM-165 & 2.79 & $6.36^{*}$ & 1.81 & $4.83^{*}$ & 10.06 & 1.42 & 10.80 & 10.36 \\
\hline 27 & CMS-89-1 X PSMOO-108 & -1.68 & 1.73 & -2.17 & 0.74 & $11.99 *$ & 3.20 & -7.60 & -7.97 \\
\hline 28 & CMS-89-1 X PSFRMM-140 & 0.00 & 3.47 & -0.72 & 2.23 & $13.18^{*}$ & 4.30 & -4.40 & -4.78 \\
\hline 29 & CMS-89-1 X MRHA-2 & 0.00 & 3.47 & -0.72 & 2.23 & 7.83 & -0.63 & 0.00 & -0.40 \\
\hline 30 & CMS-89-1 X AKSF-345 & 0.00 & 3.47 & -0.36 & 2.60 & $16.18 * *$ & 7.06 & 25.60 ** & $25.10 * *$ \\
\hline 31 & CMS-89-1 X AKSF-12 & -3.91 & -0.58 & -2.17 & 0.74 & 5.99 & -2.33 & $18.40 * *$ & $17.93 * *$ \\
\hline 32 & CMS-89-1 X CSFR-99 & -0.56 & 2.89 & -1.08 & 1.86 & 3.77 & -4.38 & $17.60 * *$ & $17.13 * *$ \\
\hline & CD@ 5\% & 3.18 & 3.18 & 3.27 & 3.27 & 16.09 & 16.09 & 1.89 & 1.89 \\
\hline & CD@ $1 \%$ & 4.23 & 4.23 & 4.35 & 4.35 & 21.39 & 21.39 & 2.51 & 2.51 \\
\hline
\end{tabular}

Contd... 


\begin{tabular}{|c|c|c|c|c|c|c|c|c|c|}
\hline \multirow[t]{2}{*}{$\begin{array}{l}\text { Sr. } \\
\text { no }\end{array}$} & \multirow[t]{2}{*}{ Hybrids } & \multicolumn{2}{|c|}{$\begin{array}{l}\text { Seed filling } \\
\text { Percent (\%) }\end{array}$} & \multicolumn{2}{|c|}{100 seed weight (g) } & \multicolumn{2}{|c|}{$\begin{array}{c}\text { Volume } \\
\text { Weight }(\mathrm{g} / \mathbf{1 0 0 m \mathrm { m } )}\end{array}$} & \multicolumn{2}{|c|}{ Hull content (\%) } \\
\hline & & $\mathrm{SC} 1$ & $\mathrm{SC} 2$ & $\mathrm{SC} 1$ & $\mathrm{SC} 2$ & $\mathrm{SC} 1$ & $\mathrm{SC} 2$ & $\mathrm{SC} 1$ & SC2 \\
\hline 1 & CMS-234 X NSH-312 & 1.24 & 3.30 & 6.06 & 6.48 & 3.73 & 6.20 & $-17.08 * *$ & $-13.66^{*}$ \\
\hline 2 & CMS-234XPSCIM165 & 5.52 & 7.67 & 5.03 & 5.44 & 0.39 & 2.78 & -4.43 & -0.50 \\
\hline 3 & CMS-234 X PSMOO-108 & 6.83 & 9.01 & 3.68 & 4.08 & 5.87 & 8.39 & $-13.45^{*}$ & -9.98 \\
\hline 4 & MS-234 X PSFRMM - 140 & -0.36 & 1.69 & 7.94 & 8.35 & 4.82 & 7.31 & $-17.49 * *$ & $-14.09 * *$ \\
\hline 5 & CMS-234 X MRHA-2 & 5.82 & 7.98 & $-19.68 *$ & $-19.37 * *$ & -2.72 & -0.41 & $11.13^{*}$ & $15.71^{* *}$ \\
\hline 6 & CMS-234 X AKSF-345 & 1.74 & 3.82 & 1.48 & 1.88 & $11.29 *$ & $13.94 * *$ & -3.02 & 0.98 \\
\hline 7 & CMS-234 X AKSF-12 & 2.56 & 4.66 & -3.29 & -2.91 & 6.19 & 9.44 & $-16.60 * *$ & $-13.16^{*}$ \\
\hline 8 & CMS-234 X CSFR-99 & 2.56 & 4.65 & -0.65 & -0.26 & 0.46 & 2.85 & $-13.78 * *$ & -10.23 \\
\hline 9 & CMS-62 X NSH-312 & 3.88 & 6.66 & $-15.42 * *$ & $-15.09 * *$ & $15.18^{* *}$ & $17.92 * *$ & 6.20 & $10.58^{*}$ \\
\hline 10 & CMS-62 X PSCIM-165 & 3.75 & 5.87 & -0.32 & 0.06 & 17.48 & $10.04^{*}$ & 0.15 & 3.97 \\
\hline 11 & CMS-62 X PSMOO-108 & 1.09 & 3.16 & $11.68^{* *}$ & $12.11^{*}$ & 1.04 & 3.44 & $-18.04 * *$ & $-14.66^{* *}$ \\
\hline 12 & CMS-62 X PSFRMM-140 & -0.01 & 2.03 & $-9.55^{*}$ & -9.20 & $17.73 * *$ & $20.53 * *$ & $-13.94 * *$ & -10.39 \\
\hline 13 & CMS-62 X MRHA-2 & 4.86 & 7.00 & $-13.03 * *$ & $-12.69 * *$ & $17.91 * *$ & $20.72 * *$ & $-18.52 * *$ & $-15.16^{* *}$ \\
\hline 14 & CMS-62 X AKSF-345 & 2.40 & 4.49 & -2.02 & -2.14 & 8.43 & $11.01^{*}$ & $-22.91 * *$ & $-19.74 * *$ \\
\hline 15 & CMS-62 X AKSF-12 & 5.93 & 8.09 & 5.16 & 5.57 & $21.86^{* *}$ & $24.76^{* *}$ & $-22.63 * *$ & $-19.45 * *$ \\
\hline 16 & CMS-62 X CSFR-99 & 0.25 & 2.30 & 5.42 & 5.83 & $19.96^{* *}$ & $22.81 * *$ & $-23.71 * *$ & $-20.56 * *$ \\
\hline 17 & CMS-343 X NSH-312 & 3.45 & 5.57 & 3.94 & 4.34 & 5.90 & 8.42 & $-23.10 * *$ & $-19.93 * *$ \\
\hline 18 & CMS-343 X PSCIM-165 & -1.98 & 0.02 & -2.00 & -1.62 & $14.46^{* *}$ & $17.18 * *$ & -8.39 & -4.62 \\
\hline 19 & CMS-343 X PSMOO-108 & 9.43 & $11.67 *$ & -1.42 & -1.04 & $23.54 * *$ & $26.47 * *$ & $-18.05 * *$ & $-14.68 * *$ \\
\hline 20 & $\begin{array}{l}\text { CMS-343 X PSFRMM- } \\
140\end{array}$ & $9.93 *$ & $12.17 *$ & -2.97 & -2.59 & $19.68^{* *}$ & $22.53 * *$ & $-20.42 * * *$ & $-17.14 * *$ \\
\hline 21 & CMS-343 X MRHA-2 & 6.15 & 8.31 & 6.19 & 6.61 & 6.36 & 8.89 & $-14.68 * *$ & $-11.17 *$ \\
\hline 22 & CMS-343 X AKSF-345 & 2.49 & 4.56 & -0.26 & 0.13 & $16.56^{* *}$ & $19.34 * *$ & -5.30 & -1.40 \\
\hline 23 & CMS-343 X AKSF-12 & 6.90 & 9.08 & -7.10 & -6.74 & $15.69 * *$ & $18.44 * *$ & -5.14 & -1.23 \\
\hline 24 & CMS-343 X CSFR-99 & 3.71 & 5.83 & 6.65 & 7.06 & -2.18 & 0.14 & $-17.12 * *$ & $-13.71 *$ \\
\hline 25 & CMS-89-1 X NSH- 13 & $10.60^{*}$ & $12.86^{*}$ & -0.90 & -0.52 & 5.09 & 7.59 & $-15.39 * *$ & $-11.90^{*}$ \\
\hline 26 & CMS-89-1 XPSCIM-165 & 9.34 & $11.57^{*}$ & 3.42 & 3.82 & 6.86 & 9.38 & -6.68 & -2.84 \\
\hline 28 & CMS-89-1 X PSMOO-108 & 4.54 & 6.67 & 8.13 & 8.55 & 5.70 & 8.21 & 7.10 & $11.51^{*}$ \\
\hline 29 & $\begin{array}{l}\text { CMS-89-1 X PSFRMM- } \\
140\end{array}$ & -0.36 & 1.67 & $17.03^{* *}$ & $17.49 * *$ & 6.41 & 8.94 & $-20.02 * *$ & $-16.72 * *$ \\
\hline 30 & CMS-89-1 X MRHA-2 & 3.51 & 5.67 & $9.81 *$ & $10.23^{*}$ & 4.57 & 7.06 & $-15.39 * *$ & $-11.90 *$ \\
\hline 31 & CMS-89-1 X AKSF-345 & -0.02 & 2.02 & 0.19 & 0.58 & $10.32 *$ & $12.95 * *$ & $-15.38 * *$ & $-11.81 *$ \\
\hline 32 & CMS-89-1 X AKSF-12 & -1.75 & 0.25 & 4.06 & 4.47 & 4.64 & 7.13 & $12.72 * *$ & $17.36^{* *}$ \\
\hline & CMS-89-1 XCSFR-99 & 5.67 & 7.83 & -4.06 & -3.69 & $11.23^{*}$ & $13.87 * *$ & -7.40 & -3.59 \\
\hline & CD @ 5\% & 7.99 & 7.99 & 0.47 & 0.47 & 4.03 & 4.03 & 2.76 & 2.76 \\
\hline & CD@ $1 \%$ & 10.63 & 10.63 & 0.63 & 0.63 & 5.35 & 5.35 & 3.67 & 3.67 \\
\hline
\end{tabular}

Contd... 


\begin{tabular}{|c|c|c|c|c|c|}
\hline \multirow[t]{2}{*}{$\begin{array}{l}\text { Sr. } \\
\text { no }\end{array}$} & \multirow[t]{2}{*}{ Hybrids } & \multicolumn{2}{|c|}{$\begin{array}{l}\text { Seed yield } \\
\text { per plant (g) }\end{array}$} & \multicolumn{2}{|c|}{ Oil content (\%) } \\
\hline & & $\mathrm{SC} 1$ & $\mathrm{SC} 2$ & SC1 & $\mathrm{SC} 2$ \\
\hline 1 & CMS-234 X NSH-312 & -2.82 & 7.10 & 3.38 & 6.99 \\
\hline 2 & CMS-234X PSCIM165 & 2.41 & 12.87 & 1.69 & 5.24 \\
\hline 3 & CMS-234 X PSMOO-108 & $21.92 * *$ & $34.42 * *$ & $7.88^{*}$ & $11.65 * *$ \\
\hline 4 & CMS-234 X PSFRMM - 140 & $25.96^{* *}$ & $38.81^{* *}$ & $8.26^{*}$ & $12.04 * *$ \\
\hline 5 & CMS-234 X MRHA-2 & -0.58 & 9.56 & $7.13^{*}$ & $10.87 * *$ \\
\hline 6 & CMS-234 X AKSF-345 & 12.84 & $24.36^{* *}$ & $12.66^{* *}$ & $16.60 * *$ \\
\hline 7 & CMS-234 X AKSF-12 & -6.91 & 2.59 & 0.19 & 3.69 \\
\hline 8 & CMS-234 X CSFR-99 & -9.51 & -0.27 & 4.41 & $8.06^{*}$ \\
\hline 9 & CMS-62 X NSH-312 & 3.58 & 14.16 & 0.56 & 4.08 \\
\hline 10 & CMS-62 X PSCIM-165 & 9.44 & $20.62 *$ & 0.00 & 3.50 \\
\hline 11 & CMS-62 X PSMOO-108 & -0.78 & 9.35 & 0.00 & 3.50 \\
\hline 12 & CMS-62 X PSFRMM-140 & -7.87 & 1.53 & 1.03 & 4.56 \\
\hline 13 & CMS-62 X MRHA-2 & -8.15 & 1.22 & 0.84 & 4.37 \\
\hline 14 & CMS-62 X AKSF-345 & -5.02 & 4.67 & 1.50 & 5.05 \\
\hline 15 & CMS-62 X AKSF-12 & 1.99 & 12.40 & 1.88 & 5.54 \\
\hline 16 & CMS-62 X CSFR-99 & -2.53 & 7.41 & -1.88 & 1.55 \\
\hline 17 & CMS-343 X NSH-312 & 10.37 & $21.64^{*}$ & 6.85 & $10.58^{*}$ \\
\hline 18 & CMS-343 X PSCIM-165 & $17.50^{*}$ & $29.49 * *$ & 0.19 & 3.69 \\
\hline 19 & CMS-343 X PSMOO-108 & 15.17 & $26.93 * *$ & 6.38 & $10.10^{*}$ \\
\hline 20 & CMS-343 X PSFRMM-140 & 14.70 & $26.40^{* *}$ & 2.35 & 5.92 \\
\hline 21 & CMS-343 X MRHA-2 & -4.80 & 4.92 & $9.94 * *$ & $13.79 * *$ \\
\hline 22 & CMS-343 X AKSF-345 & $17.33^{*}$ & $29.03 * *$ & 6.29 & $10.00 * *$ \\
\hline 23 & CMS-343 X AKSF-12 & $15.33^{*}$ & $27.10^{* *}$ & $10.69^{* *}$ & $14.56 * *$ \\
\hline 24 & CMS-343 X CSFR-99 & 10.16 & $21.41^{*}$ & 4.88 & $8.54 *$ \\
\hline 25 & CMS-89-1 X NSH- 312 & 14.27 & $25.93 * *$ & -0.75 & 2.72 \\
\hline 26 & CMS-89-1 X PSCIM-165 & $18.43^{*}$ & $30.51^{* *}$ & -4.88 & -1.75 \\
\hline 27 & CMS-89-1 X PSMOO-108 & $31.37 * *$ & $44.78 * *$ & -2.72 & 0.68 \\
\hline 28 & CMS-89-1 X PSFRMM-140 & $20.79 * *$ & $33.12 * *$ & 0.19 & 3.69 \\
\hline 29 & CMS-89-1 X MRHA-2 & 8.34 & $19.39^{*}$ & -2.35 & 1.07 \\
\hline 30 & CMS-89-1 X AKSF-345 & $20.94^{* *}$ & $33.28^{* *}$ & 1.88 & 5.44 \\
\hline 31 & CMS-89-1 X AKSF-12 & -1.93 & 8.08 & 4.97 & $8.64 *$ \\
\hline \multirow[t]{3}{*}{32} & CMS-89-1 X CSFR-99 & $24.59 * *$ & $37.31 * *$ & 1.59 & 5.15 \\
\hline & CD@ 5\% & 4.18 & 4.18 & 2.48 & 2.48 \\
\hline & CD@ $1 \%$ & 5.56 & 5.56 & 3.30 & 3.30 \\
\hline
\end{tabular}


The cross CMS-343 x CSFR-99 showed significant heterosis for days to 50 per cent flowering $(-8.94,-5.78)$, head diameter (20.80, 20.32) and hull content (-17.12, -13.71) while the cross CMS-62 x AKSF-12 for hull content $(-16.60,-13.16)$ and volume weight (21.86.24.76). Further, the cross CMS-62 x PSCIM-165 showed significant heterosis for days to maturity $(-6.86,-4.09)$ while cross CMS-343 x MRHA-2 for days to 50 per cent flowering (-10.60, -6.94), days to maturity ($7.22,-6.46)$, hull content $(-14.68,-11.17)$ and oil content $(9.94,13.79)$ over the two standard checks (Phule Raviraj and MDSFH-411). The cross CMS-89-1 x NSH-312 and CMS-343 x PSMOO-108 showed significant heterosis for seed yield and oil content over the standard check MDSFH-411. The most promising hybrids identified were CMS-343 x PSMOO108, CMS-89-1 x NSH-312, CMS-62 x AKSF-12 and CMS-343 x MRHA-2 which showed high per se performance comprising significant standard heterosis for seed yield and yield contributing attributes in desirable direction. These hybrids can be recommended for commercial cultivation after proper testing. Similar results were found by Giriraj and Virupakshappa., (1986), Govindaraju (1986), Sugoor (1992), Chidambaram and Sundershan, (1990), Madrap and Makane (1993), Putt (1996), Naresh et. al., (1996), Dedio (1992), Doddamani et. al., (1997), Gangappa et. al., (1997), Nehru et. al., (2000), Mohan Rao (2001), Latha et. al., (2005), Thombare et. al., (2007), Chigeza et. al., (2008), Karasu et.al., (2010), Manivannan et. al., (2011), Deshmukh and Bhosale, (2018), Lakshman et. al., (2018).

\section{References}

Chidambaram, S. and Sundarshan, N., 1990. Correlation between yield and yield components in sunflower (Helianthus annuus L.) Madras Agric. J. 77: 406407.
Chigeza, G., Shanahan, P., Savage, M. J., Mashingaidze K., 2008. Heterosis for yield and oil content of sunflower lines developed from bi-parental populations. Proc. $17^{\text {th }}$ International Sunflower Conference, Córdoba, Spain.

Deshmukh, Y.V. and Bhosale, S.V., 2016. Heterosis study for yield and oil content in sunflower. International Journal of Tropical Agriculture. 34(7):2167-2172.

Dedio, W., 1992. Performance comparison of single and three way crosses in sunflower. Can. J. PI. Sci., 72: 431434.

Doddamani, I.K, Patil S.A and Ravikumar R.L., 1997. Relationship of autogamy and self fertility with seed yield components in sunflower. Helia, 20: 95-102.

Gangappa, E., Channakrishniah, K.M., Ramesh, S. and Harini, A.S., 1997. Studies on combining ability in sunflower. Helia, 27:73-84.

Govindaraju, T.A., 1986. Evaluation of sunflower (Heliathus annuus L.) inbreeds by top cross analysis. M. Sc. (Agri.) Thesis, Univ.Agric. Sci. Bangalore (India)

Giriraj, K. and Virupakshappa K., 1986. Heterotic effects for seed yield and component characters in sunflower (Helianthus annuus L.).over seasons. Proc. of $13^{\text {th }}$ Int. Sunflower Conference, Pisa, Italy, 8-10th September

Karasu A., Mehmet O., Sincik M., Goksoy A.T. and Tarun Z., 2010. Combining ability and heterosis for yield and yield components in sunflower. Nat. Bot. Hort. Agrobot. Cluj., 38 (3): 541-542.

Kinman. M.L., 1970. New development in USDA and State Experimentation, Sunflower breeding programme. In: Proc. of the fourth Int. Sunflower 
Conference, Memphis Tenessa.pp 181183

Latha K.M., Vishnuvardhan Reddy A. and Devasenamma V. 2005. Hybrid vigour and combining ability in sunflower, (Helianthus annuus L.) hybrids involving CMS lines. J. Oilseed Res., 23 (2): 154-156.

Lakshman, S. S., Chakraborty, N.R., Godke, M. K., Kole, P. C., 2018. Studies on heterosis and heterobeltiosis for seed yield and yield attributing traits of sunflower (Helianthus annuus L.) in high saline soil of West Bengal. $J$. Crop and Weed 14(1): 90 - 98.

Leclereq, P., 1969. Line sterile cytoplasmique chezktournesol. Ann. Amelior Planta, 12: 99-106.

Mohan Rao, A., 2001. Heterosis is a function of genetic divergence in sunflower (Helianthus annuus L.). Ph. D Thesis, Acharya N.G. Ranga Agriculture University, Hyderabad, p. 208.

Madrap, I.A. and Makne, V.G., 1993. Heterosis in relation to combining ability effect and phenotypic stability in sunflower. J. Agric. Sci. 63(8): 484488.

Manivannan, N., Anandan, T., Chandirakala,
R., Vindhiyavarman, P., Muralidharm, V., Genesmurthy, K., 2011. New male sterile lines in sunflower (Helianthus annuus L.). Elec. J. Pl. Breed., 2(1): 58-61.

Naresh, R., Channakrishnaiah, K.M. and Gangappa E., 1996. Heterosis in single cross and three way cross hybrids of sunflower. Mysore J. Agric. Sci., 30:197-203.

Nehru, S. D., Manjunath, A. and Basavarajaiah, D., 2000. Extent of heterosis for seed yield and oil content in sunflower. Karnataka J. Agric. Sci., 13(3): 718-720.

Putt Eric D. 1996. Heterosis, combining ability and predicted synthetic from a diallel cross in sunflowers (Helianthus annuиs L.). Canadian journal of plant science 46 (1): 59-67.

Sugoor R.K., 1992. Heterosis and combining ability of induced mutant restorer lines in sunflower (Helianthus annuus L.). M. Sc. (Agri.) Thesis. Univ. Agric. Sci. Dharwad (India).

Thombare,V., Makane, V.G. and Borgaonkar,S.B., 2007. Combining ability studies in sunflower. Int. $J$. Plant. Sci. (2): 133-136.

\section{How to cite this article:}

Ramaraju, D., A. B. Rajguru, H. J. Rajput and Nimbalkar, R. D. 2019. Heterosis Studies in Sunflower (Helianthus annuus L.). Int.J.Curr.Microbiol.App.Sci. 8(09): 2155-2161. doi: https://doi.org/10.20546/ijcmas.2019.809.249 